\title{
Taller regional de utilización de resultados de investigación operativa para incrementar el acceso, la calidad e integración de los servicios anticonceptivos en América Latina y el Caribe
}

M. Estela Rivero-Fuentes

Antonieta Martin

Population Council

Follow this and additional works at: https://knowledgecommons.popcouncil.org/departments_sbsr-rh

Part of the Demography, Population, and Ecology Commons, International Public Health Commons, Public Health Education and Promotion Commons, and the Women's Health Commons

How does access to this work benefit you? Let us know!

\section{Recommended Citation}

Rivero-Fuentes, M. Estela and Antonieta Martin. 2008. "Taller regional de utilización de resultados de investigación operativa para incrementar el acceso, la calidad e integración de los servicios anticonceptivos en América Latina y el Caribe," Informe Final de FRONTERAS. Washington, DC: Population Council. 
Taller Regional de utilización de resultados de investigación operativa para incrementar el acceso, la calidad e integración de los servicios anticonceptivos en América Latina y el Caribe

\title{
Fronteras de la Salud Reproductiva (FRONTERAS), Population Council
}

\author{
M. Estela Rivero-Fuentes \\ Antonieta Martin
}

\begin{abstract}
Abril, 2008
Este estudio fue posible gracias al apoyo generoso del pueblo de los Estados Unidos, a través de la Agencia para el Desarrollo Internacional de los Estados Unidos (USAID), bajo los términos del Acuerdo de Cooperación No. HRNA-00-98-00012-00, proyecto \# 5800 53117. Los contenidos son responsabilidad de FRONTERAS y no necesariamente reflejan los puntos de vista de USAID o del Gobierno de los Estados Unidos.
\end{abstract}




\section{Resumen}

En octubre de 2007, el Programa Fronteras de la Salud Reproductiva (FRONTERAS) realizó un taller regional de tres días en La Antigua, Guatemala para difundir los resultados de sus proyectos de investigación operativa. Al taller asistieron 60 participantes de 11 países: Bolivia, República Dominicana, Ecuador, El Salvador, Guatemala, Haití, Honduras, México, Nicaragua, Perú y de los Estados Unidos. Entre los participantes había proveedores de servicios de salud, gerentes de programas, directores de organizaciones no gubernamentales (ONG), representantes de contratos bilaterales como USAID, UNFPA, UNDP, así como de representantes de los ministerios de salud, institutos de seguridad social y directores de organizaciones profesionales.

Durante el taller los participantes escucharon los avances en la metodología anticonceptiva y escucharon las estrategias efectivas que probó FRONTERAS para incrementar el acceso al DIU y la vasectomía, la integración de servicios de planificación familiar con los servicios en el posparto, posaborto y prevención de transmisión del VIH de madre a hijo. Se presentaron la Estrategia de Consejería Balanceada (ECB), y la Detección Sistemática de Necesidades de Salud Reproductiva. Los investigadores hablaron sobre los resultados de los estudios, los gerentes de los programas explicaron cómo se usaban los instrumentos y se habló de la implementación de las intervenciones. Esta combinación de perspectivas transmitieron de manera exitosa el mensaje de que las intervenciones no eran difíciles de aplicar, que podían tener gran impacto y como escribió uno de los participantes en la evaluación: "los pequeños cambios pueden llevar a grandes resultados".

Los participantes en el taller recibieron materiales desarrollados por FRONTERAS y otras instituciones para coadyuvar en los esfuerzos de integración. Los materiales distribuidos son:

a) Tres CDs. Uno fue preparado especialmente para el taller y contiene todos los informes en español de algunos informes de FRONTERAS, resúmenes de investigación operativa, manuales, ayudas de trabajo y los criterios de elegibilidad médica para el uso de métodos anticonceptivos. Este CD también tiene una compilación de artículos científicos y ayudas de trabajo para anticoncepción posparto; y un kit de herramientas sobre el DIU. El segundo CD. El Segundo CD se titula "Mejorando la calidad de los servicios de salud reproductiva. 1998-2005," y el tercero, es "La biblioteca electrónica de Fronteras de la salud reproductiva. 1990-1999."

b) La traducción al español del documento "Las preguntas más frecuentes sobre anticoncepción" de Family Health International (FHI).

c) Una versión en español del manual de detección sistemática de necesidades de salud reproductiva y ejemplos de algoritmos usados en América Latina.

d) La versión en español de la Estrategia de Consejería Balanceada con sus tarjetas de métodos, folletos y algoritmo.

Los participantes prepararon planes para poner en práctica lo aprendido en el taller. Siete países hicieron planes (Bolivia, República Dominicana, Ecuador, El Salvador, Guatemala, Honduras y Nicaragua). Otros propusieron organizar un foro electrónico para continuar hablando sobre los 
temas que se discutieron en el taller. Cuatro países dijeron que intentarían adaptar la ECB o la técnica de Detección Sistemática de Necesidades en su contexto local y tres propusieron reforzar la integración de los servicios de planificación familiar y la prevención de la transmisión del VIH de la madre al hijo.

Las evaluaciones finales del taller que hicieron 23 participantes, mostraron que el taller había sido muy útil para su trabajo. Y cuando se les preguntó qué les había gustado más, 57\% mencionó que la estrategia de consejería y 52\% mencionaron que el intercambio de experiencias. Después del taller, el Population Council creó una liga al sitio de Internet que contiene las presentaciones del taller y los materiales distribuidos. 


\section{TABLA DE CONTENIDOS}

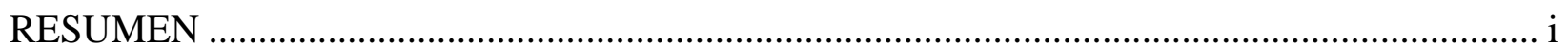

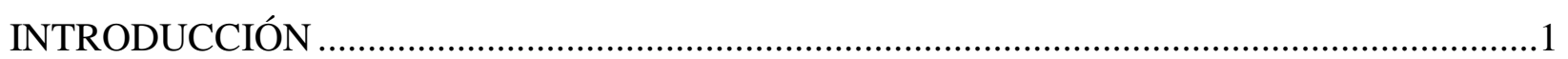

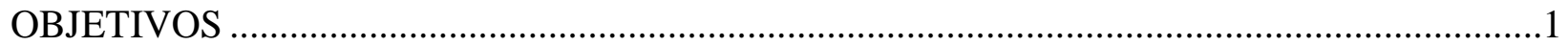

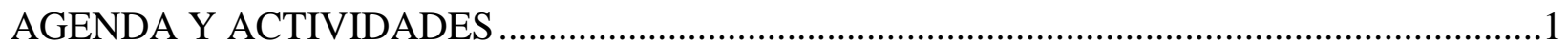

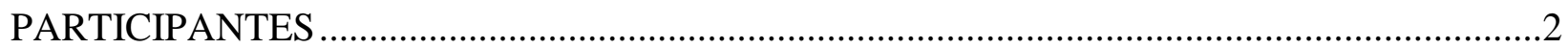

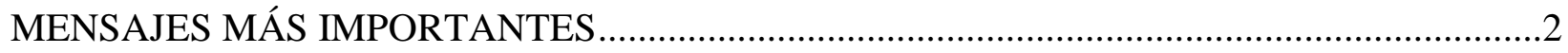

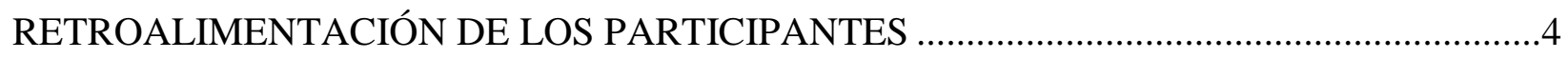

Lecciones aprendidas ..................................................................................................... 5

OBSTÁCULOSER PARA IMPLEMENTAR LAS ESTRATEGIAS E

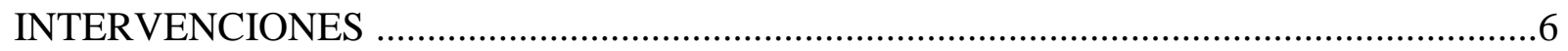

PLANES PARA IMPLEMENTAR LAS LECCIONES APRENDIDAS EN EL TALLER ..........6

APÉNDICE 1: PROYECTOS DE FRONTERAS PRESENTADOS E IMPLEMENTADOS

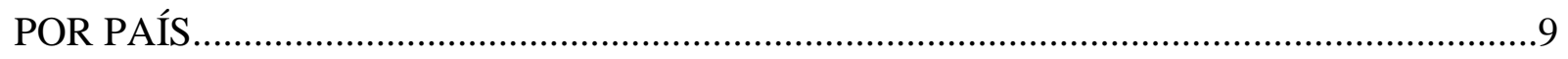

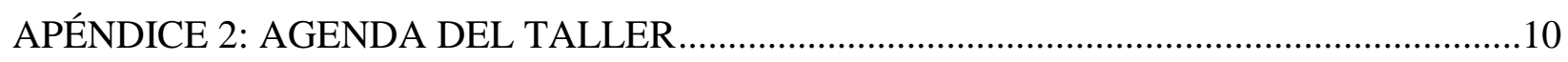

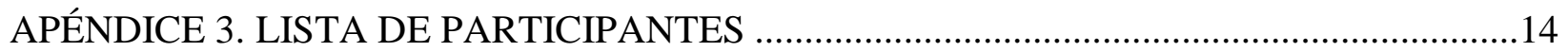

APÉNDICE 4: RESPUESTA A CUESTIONARIOS Y GUÍAS DE TRABAJO DE GRUPOS

\section{LISTA DE TABLAS}

TABLA 1: LOS TRES PRINCIPALES TEMAS QUE SE PERCIBIERON COMO LOS MÁS ÚTILES CADA DÍA

TABLA 2: LECCIONES MÁS IMPORTANTES DE LAS SESIONES DE GRUPOS DE TRABAJO. 


\section{INTRODUCCIÓN}

En América Latina, el Programa Fronteras de la Salud Reproductiva del Population Council (FRONTERAS), financiado por USAID, es una continuación del apoyo al Programa de Investigación Operativa en Planificación Familiar y Atención Materno-Infantil para América Latina y el Caribe, INOPAL I, II, y III, todos financiados por USAID e implementados por el Population Council entre 1984 y 1998. Las estrategias y resultados presentados durante el taller vienen primordialmente de los proyectos de FRONTERAS e INOPAL en la región, pero también se presentaron resultados importantes del África Subsahariana, Asia y el Medio Oriente. En total, las presentaciones de proyectos de FRONTERAS provinieron de 23 proyectos (ver Apéndice 1) y de las experiencias adquiridas por los gerentes de programas y proveedores de servicios de salud que repitieron las estrategias en mayor escala y haciendo que las intervenciones se hicieran sostenibles

El taller enfatizó la integración de la planificación familiar con otros servicios de salud y la necesidad de hacer la detección sistemática como un punto de entrada al sistema de salud por medio de la identificación y atención de las diversas necesidades de salud reproductiva y planificación familiar. Hubo también presentaciones sobre estrategias de integración de servicios de planificación familiar, posparto, posaborto y prevención de transmisión de VIH de la madre al hijo. También se discutieron las estrategias para introducir la vasectomía, la Estrategia de Consejería Balanceada y un mayor uso del DIU.

\section{OBJETIVOS}

- Contribuir al reforzamiento de los programas de planificación familiar en la región por medio de la comunicación de las lecciones aprendidas y los materiales desarrollados.

- Incrementar el conocimiento de los proyectos apoyados por FRONTERAS en su diseño, prueba e implementación de estrategias innovadoras.

- Dar un panorama de los métodos anticonceptivos disponibles en la región, revisar los criterios de elegibilidad médica de la OMS, y la situación de los programas de planificación familiar.

\section{AGENDA Y ACTIVIDADES DEL TALLER}

Durante el primer día, los participantes escucharon sobre la situación actual de los programas de planificación familiar en América Latina y el Caribe, las estrategias para incrementar el acceso al DIU en Guatemala y Honduras, y la introducción de la vasectomía sin bisturí en los hospitales públicos de Guatemala.

En el Segundo día se habló de la minilaparatomía, la anticoncepción de emergencia, la planificación familiar con métodos naturales y sobre estrategias para integrar los servicios de 
planificación familiar en el posparto, posaborto y la prevención de transmisión de madre a hijo del VIH.

Durante el tercer día, los participantes escucharon las presentaciones sobre Detección Sistemática de Necesidades y sobre la Estrategia de Consejería Balanceada, seguida de sesiones de grupo en las que aprendieron como usar las técnicas. Antes de la clausura del taller, grupos de participantes del mismo país identificaron los temas y lecciones aprendidas que les habían resultado más útiles. Y sugirieron tres acciones concretas que les gustaría realizar para implementarlas. El programa del taller está incluido en el Apéndice 2.

\section{PARTICIPANTES}

Un total de 60 participantes de organizaciones que trabajan en salud reproductiva en América Latina y el Caribe, participaron en el taller. Las misiones de USAID y del Ministerio de Salud y Deportes de Bolivia, la República Dominicana, Ecuador, El Salvador, Guatemala, Haití, Honduras, Nicaragua y Perú ayudaron a identificar a los participantes de las organizaciones más importantes que tuvieran el potencial de usar las estrategias discutidas durante el taller. La lista de participantes, se encuentra en el Apéndice 3 y muestra que los participantes venían de los ministerios de salud, instituciones de seguro social, organizaciones no gubernamentales y agencias multilaterales.

A los presentadores se les seleccionó por su participación en los estudios de investigación operativa que se discutieron en el taller y por su experiencia en alguno de los temas expuestos. Los presentadores vinieron de:

- Asociación Guatemalteca de Mujeres Médicas (AGMM) de Guatemala

- Asociación Pro-Salud Preventiva para la Mujer Vivamos Mejor (APROVIME) de Guatemala

- Oficinas del Population Council en Bolivia, México y Nueva York

- Ipas de Carolina del Norte, EUA

- Instituto Peruano de Paternidad Responsable (INPPARES), Perú

- Ministerios de Salud de República Dominicana, Honduras, Guatemala, y México.

- UNDP de Guatemala

- EngenderHealth de Honduras

- Misión de USAID en Haití.

\section{PRINCIPALES MENSAJES DEL TALLER}

Los textos de las presentaciones están en un CD. También se creará un sitio de Internet para poner estos materiales y presentaciones a disposición del público en general. 
La situación actual de los programas de planificación familiar en América Latina y el Caribe.

- John Townsend, Population Council, EUA

Fundación Acceso Internacional a los Anticonceptivos (ICA, por sus siglas en inglés) y el Sistema Intrauterino de Levonogerstrel (LNG IUS, por sus siglas en inglés)

- John Townsend, Population Council, EUA y Klaus Brill, Bayer Schering Pharma AG

La planificación familiar en el Ministerio de Salud y Asistencia Social de Guatemala

- Carlos Morales, MSPAS, Guatemala

Expansión del acceso al DIU en Guatemala y Honduras

- Carlos Morales, Edwin Montúfar, Josefina Ajpop y Carlos Fernando Alvarado, MSPAS, Guatemala, y Alba Lidia Sánchez, EngenderHealth, Honduras

Vasectomía voluntaria sin bisturí (VVSB): Nuevas técnicas para el procedimiento y estrategias de promoción para incrementar el acceso

- Salvador Estévez, Secretaría de Salud, México, Jorge Solórzano, PNUD, Guatemala and Otto René Alvarado, MSPAS, Guatemala

Minilaparatomía posparto en un Hospital de Honduras

- José Manuel Espinal, Secretaría de Salud, Honduras

Método de Días Fijos (Collar de Cuentas de Colores) en Guatemala

- Lidia de Mazariegos, APROVIME, Guatemala

Anticoncepción desde una perspectiva de los derechos, con enfoque en anticoncepción de emergencia

- Daniel Aspilcueta, INPPARES, Perú

Anticoncepción Posaborto: Revisión de resultados de investigación operativa en América Latina y el Caribe

- Deborah Billings, IPAS, EUA

La investigación operativa y la anticoncepción posparto en América Latina y el Caribe: Revisión de resultados

- Ricardo Vernon, Population Council, México

Integración de servicios anticonceptivos en programas de prevención de transmisión de madre a hijo del VIH

- Estela Rivero, (Population Council, México), Osvaldo Lorenzo, (SESPAS, República Dominicana), Yolani Valle, (Secretaria de Salud, Honduras) y Pierre Mercier, USAID, Haiti

Detección Sistemática de Necesidades: Evidencia de sus efectos en investigación operativa

- Ricardo Vernon, Population Council, Mexico y Patricia Riveros, Population Council, Bolivia 
Calidad de atención: La Estrategia de Consejería Balanceada (ECB) y sus efectos

- Toni Martin, Population Council, México

Calidad de atención: Expansión del uso de la ECB en Guatemala

- Rossana Cifuentes, AGMM, Guatemala

\section{RESPUESTA DE LOS PARTICIPANTES}

Los participantes tuvieron varias oportunidades para comunicar lo que pensaban que eran los temas más importantes y las posibles barreras en la implementación de las lecciones aprendidas en sus propias organizaciones. Los participantes llenaron un cuestionario describiendo cuáles habían sido en su opinión los temas más importantes del día.

Se les solicitó también que escribieran una pequeña evaluación sobre qué tan integrados estaban los servicios en sus instituciones, qué áreas de integración eran prioritarias para ellos y qué intervenciones presentadas en el taller podrían implementarse en sus instituciones. En el último día, los participantes llenaron una evaluación final del taller (ver Apéndice 4).

\section{Utilidad del taller y temas más relevantes}

Veintitrés participantes llenaron el cuestionario final de evaluación; 19 dijeron que el taller fue muy útil y 4 dijeron que fue útil. Cuando se les preguntó qué temas habían sido los más útiles, $57 \%$ mencionaron que la ECB. Otros temas mencionados como útiles fue la vasectomía sin bisturí, la actualización sobre los anticonceptivos y la integración de planificación familiar en el posparto, posaborto y la prevención de la transmisión madre a hijo del VIH (ver tabla 1).

Tabla 1: Tres tópicos percibidos como los más útiles, por día del taller

\begin{tabular}{|l|l|l|l|}
\hline Rango & Día 1a & Día 2 $^{\mathbf{b}}$ & $\begin{array}{l}\text { Día 3 - evaluación } \\
\text { final }^{\mathbf{c}}\end{array}$ \\
\hline $\mathbf{1}^{\text {st }}$ & $\begin{array}{l}\text { Vasectomía sin bisturí } \\
(73 \%)\end{array}$ & $\begin{array}{l}\text { Integración de PF y atención } \\
\text { posaborto (66\%) y } \\
\text { Prevención transmisión } \\
\text { madre a hijo (66\%) }\end{array}$ & BCS (57\%) \\
\hline $\mathbf{2}^{\text {nd }}$ & $\begin{array}{l}\text { Actualización anticonceptiva } \\
(59 \%)\end{array}$ & $\begin{array}{l}\text { Anticoncepción posparto } \\
(34 \%)\end{array}$ & $\begin{array}{l}\text { Vasectomía sin bisturí } \\
(22 \%)\end{array}$ \\
\hline $3^{\text {rd }}$ & $\begin{array}{l}\text { Aumentar el acceso al DIU } \\
(51 \%)\end{array}$ & $\begin{array}{l}\text { Detección Sistemática } \\
(17 \%)\end{array}$ \\
\hline
\end{tabular}

a) Porcentajes basados en 41 respuestas. Se aceptaban respuestas múltiples.

b) Porcentajes basados en 23 respuestas. Se aceptaban respuestas múltiples.

c) Porcentajes basados en 23 respuestas. Se aceptaban respuestas múltiples.

Cuando se les preguntó qué les había gustado más sobre la dinámica del taller, la mayoría de respuestas era que se presentaron estrategias prácticas para ayudar a mejorar la integración de servicios, y la oportunidad de intercambiar experiencias (mencionado por 52\%). El intercambio ayudó a los participantes a detectar las debilidades de sus propios programas: 
"Este taller nos ha ayudado a identificar las fortalezas y debilidades en nuestros países. Es también una motivación para revisitar las experiencias exitosas y para reforzar el monitoreo y la evaluación de nuestras intervenciones."

Cuando se les preguntó qué es lo que no les había gustado del taller, la mayoría se refirió a la falta de tiempo para mayor discusión (10 de cada 14 participantes). Otros participantes dijeron que les hubiera gustado tener ejercicios en grupos:

"Todo estuvo bien, pero algunas veces no había tiempo suficiente para la discusión. También el no hacer los ejercicios de grupo limitó el intercambio de experiencias."

\section{Lecciones aprendidas}

Al final del primero y segundo días, los participantes resumieron en una oración la lección más importante del día. Los mensajes más comunes incluyeron:

A) La integración de servicios requiere de trabajo de equipo:

"El sostenimiento de los programas requiere de alianzas, ya sea entre organizaciones $o$ con la sociedad civil."

"Trabajo en equipo: desde el portero hasta el director. Todos deberían participar en la promoción y aceptación de la vasectomía."

\section{B) Pequeños cambios pueden llevar a grandes resultados.}

"Todo es posible con voluntad y capacitación. No se requieren grandes recursos para implementar intervenciones que beneficien a la mayoría."

C) Se pierden muchas oportunidades en la prestación de servicios de planificación familiar "Todas las ocasiones son buenas para ofrecer a los clientes todos los servicios de salud reproductiva."

"Deberíamos tomar acción y liderazgo en las decisiones para integrar los servicios. El compromiso debe existir en todos los niveles."

"La voluntad de los proveedores de salud es una parte central de todas las acciones."

D) Es importante aprender de las experiencias y de las prácticas basadas en evidencia.

"Aún cuando sepamos mucho sobre planificación familiar, siempre hay oportunidad de aprender de otros."

"Si compartimos nuestras experiencias, creceremos juntos." 


\section{OBSTÁCULOS QUE SE PERCIBEN PARA IMPLEMENTAR LAS ESTRATEGIAS E INTERVENCIONES DISCUTIDAS}

Durante los ejercicios de cierre al final del primero y segundo días, los participantes listaron las barreras que piensan podrían enfrentar si las intervenciones presentadas se replicaran en sus instituciones. Las barreras mencionadas para poder incrementar el acceso al DIU, la vasectomía, la integración de servicios de planificación familiar posparto y posaborto, así como la prevención de transmisión de madre a hijo del VIH, fueron las siguientes:

1) Falta de apoyo institucional. La mitad de los participantes que respondieron a los cuestionarios de retroalimentación, mencionaron que los esfuerzos para mejorar el acceso al DIU y la vasectomía y para aumentar la integración de servicios de planificación familiar con otros servicios, depende en gran medida del apoyo del Ministerio de Salud que no ha estado siempre disponible. Otros mencionaron que las organizaciones no estaban interesadas en formar alianzas.

2) Las actitudes negativas del personal y la falta de capacitación, especialmente en la inserción de DIU, vasectomías y la integración con programas de prevención de transmisión de madre a hijo. Los participantes también mencionaron que en algunas instancias, el personal capacitado no tiene la confianza suficiente para insertar DIU o realizar vasectomías.

3) Hay problemas económicos como falta de financiamiento y de métodos.

4) Percepciones culturales, mitos y miedo relacionados al DIU y la vasectomía.

5) Falta de coordinación para el financiamiento entre los programas, esto es particularmente relevante en el caso de los programas de planificación y prevención de transmisión del VIH de madre a hijo.

6) La influencia de la iglesia y de otros grupos conservadores.

\section{PLANES PARA IMPLEMENTAR LAS LECCIONES APRENDIDAS DURANTE EL TALLER}

Al finalizar el taller, los participantes formaron grupos de trabajo por país para identificar los tres temas o lecciones que consideraban más relevantes para sus países y para definir tres actividades que podrían ayudar a implementar estas lecciones (las guías que se entregaron para el trabajo de grupos están adjuntas al final del Apéndice 4). Representantes de siete países completaron este ejercicio.

La Tabla 2 resume las lecciones que los países reportaron como las más importantes. Con excepción de Ecuador, todos los países mencionaron la Estrategia de Consejería Balanceada y la Detección Sistemática de Necesidades como las más importantes. Otros temas considerados como de especial relevancia, fueron la vasectomía sin bisturí y la integración de servicios de planificación familiar y prevención de transmisión de madre a hijo del VIH. 
Tabla 2: $\quad$ Temas más importantes para los grupos de trabajo por país

\begin{tabular}{|l|c|c|c|c|c|c|c|}
\hline \multirow{2}{*}{ Tema } & \multicolumn{7}{c|}{ País } \\
\cline { 2 - 8 } & Bolivia & $\begin{array}{c}\text { Rep. } \\
\text { Dom }\end{array}$ & Ecuador & $\begin{array}{c}\text { El } \\
\text { Salvador }\end{array}$ & Guatemala & Honduras & Nicaragua \\
\hline ECB & $\square$ & $\square$ & $\square$ & $\square$ & $\square$ & $\square$ & $\square$ \\
\hline $\begin{array}{l}\text { Detección } \\
\text { Sistemática }\end{array}$ & $\square$ & $\square$ & & $\square$ & $\square$ & $\square$ & $\square$ \\
\hline $\begin{array}{l}\text { Vasectomía sin } \\
\text { bisturí }\end{array}$ & $\square$ & $\square$ & $\square$ & $\square$ & & & $\square$ \\
\hline $\begin{array}{l}\text { Integración de } \\
\text { PF/PTMH }\end{array}$ & & & & $\square$ & & & $\square$ \\
\hline $\begin{array}{l}\text { Actualización } \\
\text { metodología } \\
\text { anticonceptiva }\end{array}$ & & & $\square$ & & & & $\square$ \\
\hline $\begin{array}{l}\text { Estrategias para } \\
\text { incrementar el } \\
\text { acceso as DIU }\end{array}$ & & & & & & & $\square$ \\
\hline $\begin{array}{l}\text { Estrategias para } \\
\text { incrementar el } \\
\text { acceso a la } \\
\text { vasectomía }\end{array}$ & & & & & & & \\
\hline
\end{tabular}

Cinco países (Bolivia, Dominicana, Ecuador, El Salvador, Guatemala, Honduras y Nicaragua) mencionaron que implementarían la ECB y/o la Detección Sistemática de Necesidades, e incluyeron actividades concretas de seguimiento:

\section{Bolivia:}

a) Compartir las lecciones del taller con autoridades nacionales e institucionales.

b) Desarrollo de planes de acción.

c) Promover el intercambio de expresiones y experiencias entre instituciones y países.

\section{República Dominicana:}

a) UNFPA y el Instituto de Seguro Social organizarán una reunión conjunta para compartir los resultados del taller con autoridades nacionales e institucionales.

b) Tomar el liderazgo para crear un foro de intercambio de experiencias entre los participantes del taller, y crear alianzas entre instituciones y agencias de cooperación.

\section{Ecuador:}

a) Capacitar en la consejería usando la ECB.

b) Capacitar a los proveedores de planificación familiar en consejería para promover la minilaparatomía y vasectomía posparto.

c) Capacitar a un número mayor de proveedores para reforzar la planificación familiar posaborto.

\section{El Salvador:}

a) Compartir las lecciones aprendidas con el Comité Nacional de Seguridad Anticonceptiva $\mathrm{y}$ asociaciones profesionales. 
b) Adaptar e implementar la ECB en el Ministerio de Salud, el Instituto de Seguro Social y las ONG.

c) Adaptar e implementar la detección sistemática de necesidades en el Ministerio de Salud.

d) Continuar la capacitación en inserción de IUD, en la vasectomía sin bisturí y la minilaparatomía en el Ministerio y en el Instituto de Seguro Social.

e) Iniciar las actividades para integrar la planificación familiar con la PTMH.

\section{Guatemala:}

a) Fortalecer la consejería en planificación familiar en todos los sectores. .

b) Fortalecer la implementación de la detección sistemática y de la ECB.

\section{Honduras:}

a) Compartir los materiales y resultados de la ECB y de la detección sistemática con las autoridades del Ministerio de salud.

b) Hablar sobre la implementación de ECB y la detección sistemática con los directores de planificación familiar y VIH/SIDA en el Ministerio.

c) Desarrollar un plan de acción.

\section{Nicaragua:}

a) Compartir materiales y resultados del taller con las autoridades nacionales.

b) Organizar charlas en hospitales y clínicas para sensibilizar al personal de salud sobre la necesidad de integrar la planificación familiar con otros servicios.

c) Monitorear la implementación de las actuales intervenciones.

d) Fortalecer el programa de PTMH. 


\section{APÉNDICE 1: PROYECTOS DE FRONTERAS PRESENTADOS DURANTE EL TALLER, POR PAÍS}

\begin{tabular}{|c|c|}
\hline País & $\begin{array}{c}\text { Proyecto } \\
\end{array}$ \\
\hline Bolivia & $\begin{array}{l}\text { Investigación Operativa para mejorar los servicios en el posaborto (APA) } \\
\text { en tres hospitales de Bolivia }\end{array}$ \\
\hline Bolivia & $\begin{array}{l}\text { Asistencia técnica y capacitación al programa de Investigación Operativa } \\
\text { de PROCOSI. }\end{array}$ \\
\hline Bolivia & Asistencia técnica a Pathfinder sobre investigación operativa \\
\hline Bolivia & $\begin{array}{l}\text { Prueba de un modelo de atención durante la emergencia obstétrica y } \\
\text { servicios de planificación familiar en el sistema de salud pública de } \\
\text { Bolivia. }\end{array}$ \\
\hline Bolivia & Uso de la oferta sistemática para los servicios de salud reproductiva \\
\hline $\begin{array}{l}\text { República } \\
\text { Dominicana }\end{array}$ & $\begin{array}{l}\text { Servicios anticonceptivos en los programas posparto, posaborto y en la } \\
\text { PTMH, }\end{array}$ \\
\hline Guatemala & Disponibilidad y aceptación del DIU en Guatemala \\
\hline Guatemala & $\begin{array}{l}\text { Mejora en la elección del método de manera informada, continuidad en } \\
\text { la planificación familiar, mezcla de métodos y costo-efectividad en la } \\
\text { clínica de posparto/posaborto en el Hospital de Ginecología y Obstetricia } \\
\text { del Instituto Guatemalteco de Seguro Social (IGSS) }\end{array}$ \\
\hline Guatemala & $\begin{array}{l}\text { Incrementar el acceso a métodos anticonceptivos de larga duración } \\
\text { (DIU) en áreas rurales a través del Ministerio de Salud de Guatemala }\end{array}$ \\
\hline Guatemala & Expansión del acceso a los servicios de vasectomía en Guatemala \\
\hline Guatemala & Atención de la salud reproductiva en el periodo posparto \\
\hline Guatemala & Incrementar el uso de un modelo exitoso de consejería: Guatemala \\
\hline Guatemala & $\begin{array}{l}\text { Prueba del algoritmo de consejería y sus ayudas de trabajo y supervisión } \\
\text { para mejorar la calidad de atención en planificación familiar en los } \\
\text { centros y puestos de salud del Ministerio de Salud de Guatemala }\end{array}$ \\
\hline Haiti & $\begin{array}{l}\text { Análisis de la situación del uso de anticonceptivos en el posparto, } \\
\text { posaborto y prevención de la transmisión de madre a hijo del VIH }\end{array}$ \\
\hline Honduras & Difusión de información sobre el DIU en áreas rurales de Honduras \\
\hline Honduras & Expansión de la anticoncepción en el posparto y posaborto \\
\hline Honduras & $\begin{array}{l}\text { Expansión del rol de las auxiliares de salud en la provisión de servicios } \\
\text { de planificación familiar fase } 1 \text { y fase } 2\end{array}$ \\
\hline Honduras & $\begin{array}{l}\text { Detección sistemática como una estrategia para aumentar la integración } \\
\text { de servicios e ingresos monetarios }\end{array}$ \\
\hline India & $\begin{array}{l}\text { Detección sistemática para integrar servicios de salud reproductiva en } \\
\text { India }\end{array}$ \\
\hline Nicaragua & $\begin{array}{l}\text { Análisis de la situación en el uso de anticonceptivos en los programas de } \\
\text { posparto, posaborto y PTMH, Nicaragua }\end{array}$ \\
\hline Perú & $\begin{array}{l}\text { Impactos de una intervención diseñada para mejorar la interacción } \\
\text { cliente-proveedor en clínicas del Ministerio de Salud de Perú }\end{array}$ \\
\hline Senegal & $\begin{array}{l}\text { Uso de la Detección Sistemática para mejorar la integración de los } \\
\text { servicios de salud reproductiva en Senegal }\end{array}$ \\
\hline
\end{tabular}




\section{APÉNDICE 2: AGENDA DEL TALLER}

\begin{tabular}{|c|c|c|}
\hline \multicolumn{3}{|c|}{ Lunes, 8 de octubre de 2007} \\
\hline 19:30-21:30 & \multicolumn{2}{|l|}{ Cena de bienvenida } \\
\hline \multicolumn{3}{|c|}{ Martes, 9 de octubre de 2007} \\
\hline 07:00 - 8:00 & \multicolumn{2}{|l|}{ Desayuno } \\
\hline 08:00 - 8:45 & \multicolumn{2}{|l|}{ Registro de participantes } \\
\hline 09:00 - 09:30 & $\begin{array}{l}\text { Inauguración y presentación de participantes } \\
\text { distinguidos: } \\
\text { Pierre Mercier, USAID/Haiti } \\
\text { John Townsend, Population Council/New York }\end{array}$ & $\begin{array}{l}\text { Moderador: } \\
\text { Alejandro Silva, } \\
\text { MSPAS/Guatemala }\end{array}$ \\
\hline 09:30-10:00 & $\begin{array}{l}\text { Situación actual de los Programas de Planificación } \\
\text { Familiar en América Latina y el Caribe }\end{array}$ & $\begin{array}{l}\text { John Townsend, } \\
\text { Population Council/NY }\end{array}$ \\
\hline $10: 00-10: 15$ & \multicolumn{2}{|l|}{ Descanso } \\
\hline $10: 15-10: 30$ & Objetivos del taller & $\begin{array}{l}\text { Estela Rivero, Population } \\
\text { Council/México }\end{array}$ \\
\hline $\begin{array}{l}10: 30-11: 15 \\
11: 15-11: 30\end{array}$ & $\begin{array}{l}\text { Actualización en tecnología anticonceptiva: el DIU } \\
\text { El IUS } \\
\text { Preguntas y respuestas }\end{array}$ & $\begin{array}{l}\text { Carlos Morales, } \\
\text { MSPAS/Guatemala } \\
\text { John Townsend, } \\
\text { Population Council/NY }\end{array}$ \\
\hline 11:30-12:00 & Expansión del acceso al DIU en Guatemala & $\begin{array}{l}\text { Edwin Montúfar, } \\
\text { MSPAS/Guatemala }\end{array}$ \\
\hline $12: 00-12: 15$ & $\begin{array}{l}\text { La experiencia de una enfermera exitosa en el programa } \\
\text { de DIU en el MSPAS de Guatemala }\end{array}$ & $\begin{array}{l}\text { Josefina Ajpop and Carlos } \\
\text { Fernando Alvarado, } \\
\text { MSPAS/Guatemala }\end{array}$ \\
\hline $12: 15-12: 45$ & Expansión del acceso al DIU en Honduras & $\begin{array}{l}\text { Alba L. Sánchez, } \\
\text { EngenderHealth/Honduras }\end{array}$ \\
\hline $12: 45-13: 15$ & $\begin{array}{l}\text { Elegibilidad, listas de verificación para clientas del } \\
\text { DIU, descarte de embarazo y otras ayudas de trabajo }\end{array}$ & $\begin{array}{l}\text { Edwin Montúfar, } \\
\text { MSPAS/Guatemala }\end{array}$ \\
\hline $13: 15-14: 15$ & \multicolumn{2}{|l|}{ Almuerzo } \\
\hline
\end{tabular}




\begin{tabular}{|c|c|c|}
\hline $\begin{array}{l}14: 15-14: 45 \\
14: 45-15: 00\end{array}$ & $\begin{array}{l}\text { Actualización en tecnología anticonceptiva: Vasectomía } \\
\text { Preguntas y respuestas }\end{array}$ & $\begin{array}{l}\text { Salvador Estévez, } \\
\text { Secretaría de Salud/México }\end{array}$ \\
\hline $15: 00-15: 30$ & $\begin{array}{l}\text { Resultados del uso de un modelo sistémico para } \\
\text { introducir la vasectomía sin bisturí en hospitales y } \\
\text { maternidades de Guatemala }\end{array}$ & $\begin{array}{l}\text { Jorge Solórzano, } \\
\text { PNUD/Guatemala }\end{array}$ \\
\hline $15: 30-16: 00$ & $\begin{array}{l}\text { La experiencia de un cirujano exitoso en el programa de } \\
\text { vasectomía del MSPAS de Guatemala }\end{array}$ & $\begin{array}{l}\text { Otto René Alvarado, } \\
\text { MSPAS/Guatemala }\end{array}$ \\
\hline $16: 00-17: 30$ & \multicolumn{2}{|l|}{ Descanso } \\
\hline $16: 15-17: 30$ & $\begin{array}{l}\text { Resumen del día: } \\
\text { Identificar las lecciones más importantes del día } \\
\text { expresadas por escrito }\end{array}$ & $\begin{array}{l}\text { Moderadores: } \\
\text { Estela Rivero, Population } \\
\text { Council/México } \\
\text { Salvador Estévez, } \\
\text { Secretaría de Salud/ } \\
\text { México }\end{array}$ \\
\hline \multicolumn{3}{|c|}{ Miércoles, 10 de octubre de 2007} \\
\hline $7: 30-8: 45$ & \multicolumn{2}{|l|}{ Desayuno } \\
\hline $9: 00-10: 30$ & $\begin{array}{l}\text { Actualización en tecnología anticonceptiva: Atención } \\
\text { posparto, minilaparotomía, método de días fijos y } \\
\text { anticoncepción de emergencia }\end{array}$ & $\begin{array}{l}\text { Moderadora: } \\
\text { Deborah Billings, } \\
\text { Ipas/EUA } \\
\text { José Manuel Espinal, } \\
\text { Secretaría de Salud/ } \\
\text { Honduras } \\
\text { Lidia de Mazariegos, } \\
\text { APROVIME/Guatemala } \\
\text { Daniel Aspilcueta, } \\
\text { INPPARES/Perú }\end{array}$ \\
\hline $10: 30-11: 00$ & \multicolumn{2}{|l|}{ Descanso } \\
\hline $\begin{array}{l}11: 00-11: 45 \\
11: 45-12: 00\end{array}$ & $\begin{array}{l}\text { Anticoncepción posaborto: Revisión de resultados de } \\
\text { investigación operativa en la región } \\
\text { Preguntas y respuestas }\end{array}$ & $\begin{array}{l}\text { Deborah Billings, } \\
\text { Ipas/EUA }\end{array}$ \\
\hline $\begin{array}{l}12: 00-13: 00 \\
13: 00-13: 15\end{array}$ & $\begin{array}{l}\text { Investigación operativa en anticoncepción posparto en } \\
\text { América Latina y el Caribe: Revisión de resultados } \\
\text { Preguntas y respuestas }\end{array}$ & $\begin{array}{l}\text { Ricardo Vernon, } \\
\text { Population Council/México }\end{array}$ \\
\hline
\end{tabular}




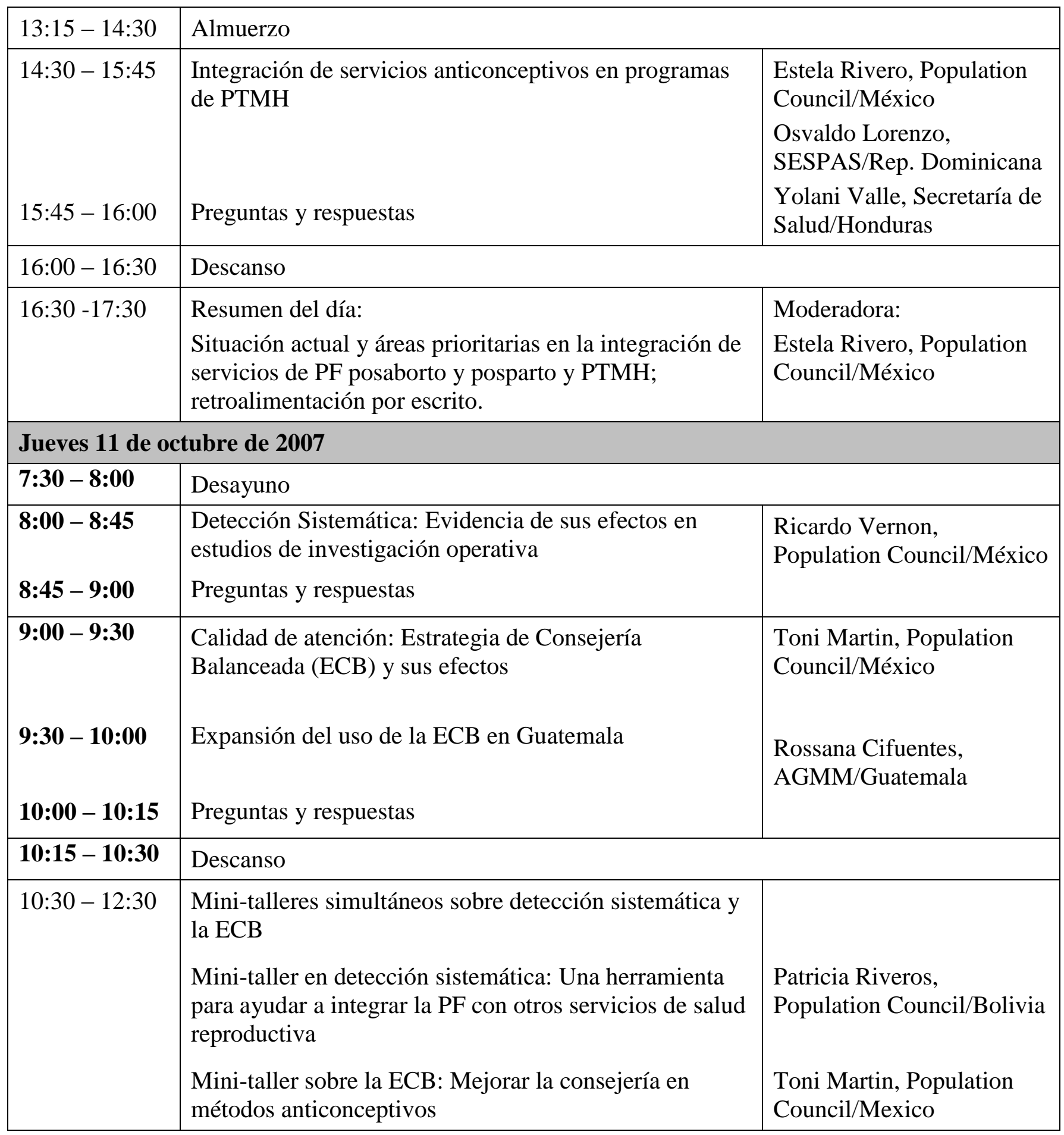




\begin{tabular}{|c|c|c|}
\hline $12: 30-13: 00$ & Grupos de trabajo por país: Planear para el futuro & \multirow{3}{*}{$\begin{array}{l}\text { Moderadora: } \\
\text { Estela Rivero, Population } \\
\text { Council/México }\end{array}$} \\
\hline $13: 00-13: 30$ & $\begin{array}{l}\text { Presentaciones de los grupos de trabajo por país y } \\
\text { conclusiones }\end{array}$ & \\
\hline $13: 30-13: 45$ & Evaluación del taller: Retroalimentación por escrito & \\
\hline $13: 45-14: 00$ & Cierre del taller & $\begin{array}{l}\text { Ricardo Vernon, } \\
\text { Population Council/México }\end{array}$ \\
\hline $14: 00-15: 00$ & \multicolumn{2}{|l|}{ Almuerzo } \\
\hline $15: 00-19: 30$ & \multicolumn{2}{|l|}{ Tarde libre } \\
\hline $19: 30-22: 30$ & $\begin{array}{l}\text { Cena para presenter el libro: Billings, Deborah, Ricardo } \\
\text { Vernon, (Comps). 2007. "Avances en la atención } \\
\text { posaborto en América Latina y el Caribe: Investigando, } \\
\text { aplicando y aprendiendo [Advances in Postabortion } \\
\text { Care in Latin America and the Caribbean: Research, } \\
\text { Implementation and Scaling-up].” Mexico, D.F.: Ipas, } \\
\text { Population Council. }\end{array}$ & $\begin{array}{l}\text { Presentadores: } \\
\text { Nadine Gasman, UNFPA/ } \\
\text { Guatemala } \\
\text { Gustavo Gutiérrez, } \\
\text { Independent practice/ } \\
\text { former Director IGSS, } \\
\text { Guatemala } \\
\text { Deborah Billings, } \\
\text { IPAS/NC } \\
\text { Ricardo Vernon, } \\
\text { Population Council/Mexico }\end{array}$ \\
\hline
\end{tabular}




\section{APÉNDICE 3. LISTA DE PARTICIPANTES}

\begin{tabular}{l}
\hline \multicolumn{1}{c}{ Bolivia } \\
\hline Dr. José Luis Alfaro \\
Gerente Técnico \\
Centro de Investigación, Estudios y \\
Servicios (CIES) \\
Dra. Carmen Estepa \\
Gerente de las Unidades Móviles de Salud \\
y Servicios \\
Centro de Investigación, Estudios y \\
Servicios (CIES) \\
Dr. Julio César Koca \\
Gerente de Planeación de Servicios de \\
Salud \\
Ministerio de Salud y Deportes \\
Lic. Patricia Riveros \\
Consultora \\
The Population Council
\end{tabular}

\section{República Dominicana}

Dra. Ana Delia Figueroa

Gerente de Servicios Clínicos

Secretaria de Estado de Salud Pública y

Asistencia Social (SESPAS)

Dr. Osvaldo A. Lorenzo

Gerente del Programa Nacional para

Reducir la Transmisión Vertical del VIH

Dirección General de Control de las ITS y SIDA (DIGECITSS)

Secretaria de Estado de Salud Pública y

Asistencia Social (SESPAS)

Dra. Luz A. Mercedes

Coordinadora Médica

Fondo de las Naciones Unidas en

Población (UNFPA)
Dra. Clavel Sánchez

Gerente de Salud Reproductiva

Instituto Dominicano de Seguros Sociales

(IDSS)

Dra. Clara I. Santillán

Coordinadora Técnica

Asociación Dominicana Pro-Bienestar de la Familia (PROFAMILIA)

Dra. Nury M. Vargas

Médica

Asociación Dominicana Pro-Bienestar de la Familia (PROFAMILIA)

Dra. Rumalda A. Vázquez

Médica

Asociación Dominicana Pro-Bienestar de la Familia (PROFAMILIA)

\section{Ecuador}

Dra. Magda C. Acosta

Médica

Centro Médico de Orientación y

Planificación Familiar (CEMOPLAF)

Dra. Tula L. Verdezoto

Directora del Centro Médico Bolívar en

Guaranda

Centro Médico de Orientación y

Planificación Familiar (CEMOPLAF)

\section{EI Salvador}

Ing. Rafael A. Avendaño

Director Ejecutivo

Asociación Demográfica Salvadoreña

(ADS) 
Lic. Claudia Lara

Enfermera del Programa de Atención

Integral de la mujer

Instituto Salvadoreño del Seguro Social

(ISSS)

Dra. L. Esmeralda Ramirez

Coordinadora Nacional del Programa de

Planificación Familiar

Ministerio de Salud y Asistencia Social

(MSPAS)

Dr. Mario A. Morales

Oficial de Salud Reproductiva

Fondo de Población de las Naciones

Unidas (UNFPA)

Dr. Carlos A. Morales

Coordinador Local, Programa de Atención

Integral a las Mujeres

Instituto Salvadoreño del Seguro Social

(ISSS)

Dra. Irma Georgina Santamaría

Coordinadora, Programa de Salud Integral

para las Mujeres

Instituto Salvadoreño del Seguro Social

(ISSS)

\section{Guatemala}

Lic. Josefina Ajpop

Enfermera profesional

Centro de Salud Patzicia

Ministerio de Salud Pública y Asistencia

Social (MSPAS)

Lic. Otto René Alvarado

Director del Hospital "Maternidad Primero

de Julio"

Ministerio de Salud Pública y Asistencia

Social (MSPAS)

Lic. Carlos Fernando Alvarado

Coordinador de IEC

Centro de Salud Patzicia
Ministerio de Salud Pública y Asistencia

Social (MSPAS)

Dra. Sandra Barahona

Médica

Alianzas/USAID

Dr. Rodrigo Barillas

Coordinador de Salud

Alianzas/USAID

Lic. Carlos Bauer

Director Ejecutivo

Asociación Pro Bienestar de la Familia de

Guatemala (APROFAM)

Dra. Rossana Cifuentes

Presidenta

Asociación Guatemalteca de Mujeres

Médicas (AGMM)

Nadine Gasman

Representante

Fondo de Población de las Naciones

Unidas (UNFPA)

Dr. Gustavo Gutiérrez

Ginecólogo, Ex-Director de Salud

Reproductiva IGSS

Lic. Lidia A. de Mazariegos

Directora

Asociación Pro-Salud Preventiva para la

Mujer Vivamos Mejor (APROVIME)

Dr. Edwin Montúfar

Asesor Técnico en Salud Reproductiva

MSPAS

Dr. Carlos René Morales

Asesor de PF del Programa Nacional de

Salud Reproductiva

Ministerio de Salud Pública y Asistencia

Social (MSPAS) 
Dr. Edwin Morales

Gerente de Servicios Médicos

Asociación Pro-Bienestar de la Familia de Guatemala (APROFAM)

\section{Lic. Blanca E. Rodríguez \\ Consultora \\ Fondo de Población de las Naciones \\ Unidas (UNDP) \\ Dr. Alejandro Silva \\ Coordinador del Programa Nacional de \\ Salud Reproductiva \\ Ministerio de Salud Pública y Asistencia \\ Social (MSPAS) \\ Dr. Jorge Solórzano \\ Director UEP \\ Programa de Desarrollo de las Naciones \\ Unidas (UNDP) \\ Dra. Berta Taracena \\ Consultora \\ Proyectos, Consultorías y Desarrollo (PROCONDE)}

\section{Haiti}

Florence D. Guillaume, Médica

Jefa Adjuntade/Directora Técnica

Management Sciences for Health (MSH)

Dr. Lucito Jeannis

Representante

JHPIEGO

Pierre Mercier, Médico, MSP

Asesor Médico Senior/Especialista en

Asistencia para el Desarrollo

Agencia para el Desarrollo Internacional

de los Estados Unidos (USAID)

\section{Honduras}

Lic. Élida R. Aguilar

Enfermera, Programa Nacional de

Atención Integral para las Mujeres

Secretaría de Salud

Dr. Manuel E. Carrasco

Jefe de Atención del Programa para

Hombres

Secretaría de Salud

Dr. Jose Manuel Espinal

Jefe de Atención de Urgencias, Hospital

Materno-Infantil

Secretaría de Salud

Dra. Flor M. Matute

Gerente Subprograma de Salud

Reproducitva

Fondo de Población de las Naciones

Unidas (UNFPA)

Lic. Alba Lidia Sánchez

Directora de País

EngenderHealth, Honduras

Dr. Manuel A. Sandoval

Director Médico

ASHONPLAFA

Lic. Yolany C. Valle

Coordinadora Departamental del Programa de Prevención de Transmisión de Madre a Hijo del VIH,

Secretaría de Salud

\section{Mexico}

Dr. Salvador Estévez

Capacitador Nacional y Certificador del

Programa de Vasectomía

Centro Nacional de Equidad de Género y

Salud Reproducitva

Secretaría de Salud 
Lic. Antonieta Martin

Oficial Regional de Comunicación

Population Council/FRONTERAS

Susana Medina

Asistente Administrativa

Population Council/FRONTERAS

Dra. Estela Rivero

Asociada del Programa

Population Council/FRONTERAS

Dr. Ricardo Vernon

Director Regional

Population Council/FRONTERAS

\section{Nicaragua}

Dra. Dinorah Corea

Coordinadora de Servicios de la

Organización

Ministerio de Salud

Dra. M. Fabiola González

Médica

Pro Mujer Nicaragua

Dr. Luis Manuel Urbina

Safe Motherhood Advisor

Healthcare Improvement Project/USAID

Nicasalud - Famisalud/USAID

Dr. Alejandro Ortiz

Medical Care Coordinator

Ministerio de Salud

Dra. Sonia M. Picado

Médica

Gerente de Calidad y Monitoreo

Centro de Mujeres Ixchen
Especialista en Salud Sexual y

Reproductiva

\section{Perú}

Dr. Daniel Aspilcueta

Director Ejecutivo

Instituto Peruano de Paternidad

Responsable (INPPARES)

\section{Estados Unidos}

Deborah L. Billings, PhD

Senior Associate, Research and Evaluation Ipas

John W. Townsend, Ph.D.

Reproductive Health Program Director

The Population Council 


\section{APÉNDICE 4: CUESTIONARIOS DE RETROALIMENTACIÓN Y GUÍAS PARA LOS GRUPOS DE TRABAJO}

Cuestionario de retroalimentación

Martes 9 de octubre de 2007 y

Miércoles 10 de octubre de 2007

Por favor conteste las siguientes preguntas antes de salir del salón de reuniones por la tarde. Esto no le tomará más de tres minutos.

1. ¿Cuáles de los temas discutidos hoy fue el más interesante o útil para su trabajo?

2. Resuma en una frase, un mensaje o lo que aprendió hoy

3. Tiene alguna sugerencia para incrementar el impacto de las lecciones y experiencias de las que se habló hoy para su país u organización?

Muchas gracias y nos veremos mañana 


\section{Cuestionario final de retroalimentación \\ Jueves 11 de octubre de 2007}

Este cuestionario nos ayudará a conocer su opinión general sobre el taller y qué podríamos para mejorar los futuros talleres e intentos de compartir nuestras experiencias. Responder a las siguientes preguntas no le tomará más de 5 minutos y sus respuestas serán muy útiles para nosotros.

1. En general, ¿diría usted que el taller fue muy útil, algo útil o no fue útil para su trabajo?

2. ¿Qué fue lo que más le gusto del taller?

3. ¿Qué fue lo que menos le gustó?

4. ¿Diría usted que las experiencias y lecciones discutidas fueron útiles para mejorar los servicios de planificación familiar en su país u organización?

5. De los temas y experiencias discutidas, ¿hay alguna en particular que sea relevante para su país u organización?

6. Tiene usted planes para compartir las lecciones del taller cuando esté en su país? ¿Cuáles serían los planes?

\section{¡Gracias!}




\title{
Guide 1
}

Guide for working groups 1: Identification of the day's most important lessons and of the interventions that can be useful in your program

\author{
Tuesday, October 9, 2007
}

Note: Instructions for dividing into groups and group dynamics are omitted as in the end, participants were asked to write an answer to the guiding questions individually.

During this exercise, each group should identify:

- Which concrete actions can be taken to increase access to the IUD in their country or organization

- Which obstacles may exist for these actions

- Which concrete actions can be taken to increase access to no-scalpel vasectomy in hospitals and maternity wards

- Which obstacles may exist for these actions 


\title{
Guide 2
}

Guide for working groups 2: Current situation and recommendations to improve the integration of family planning and postpartum, postabortion and PMTCT services

\author{
Wednesday, October 10
}

Note: Instructions for dividing into groups and group dynamics are omitted as in the end, participants were asked to write an answer to the guiding questions individually.

Participants will discuss the following issues:

- How integrated are family planning services with postabortion, postpartum and PMTCT services in your country or organization?

- What should be the priority in these integration efforts?

- Which of the integration strategies discussed today would be feasible in your organization?

- Which would be the obstacles to these strategies? 


\section{Guide 3}

\section{Guide for working groups 3: Planning for the future}

Thursday, October 11, 2007

This last session has the goal of allowing participants from the same country to discuss whether and how the lessons and topics discussed during the workshop may be of use to them, and to propose concrete actions for their implementation.

Participants from the same country should get together and form a working group. Each group will have a special place to work, with a blackboard and markers to support their work. Each group has an hour to identify:

- Which of the topics and lessons discussed during the program is of most use for their program

- $\quad$ Three concrete actions to put the lessons learned into practice that they will take when they go back to their country

We will gather again in the meeting room at 15:45. At that time, each group will present, in 5 minutes, the lessons that they identified as a priority and their action plans. 